\title{
The Application of the Smart Technique in the Construction and Management of Urban Park Green Spaces
}

\author{
Yichuan Zhang ${ }^{1 *}$ and Yan $\mathrm{Li}^{2}$ \\ 1. School of Horticulture and Landscape Architecture, Henan Institute of \\ Science and Technology, Xinxiang 453003, China; \\ 2. Henan Technical College of Construction, Zhengzhou 450064, China \\ zhangyichuan2002@163.com \\ *Corresponding Author
}

\begin{abstract}
Urban park green space is an integral part of urban public green space. The smart technique can improve the efficiency of the construction and management of urban parks. This research combines the life cycle of park green space with users' requirement, and establishes an overall frame of a system in which smart technique is applied to the park green space, namely a layered structure which consists of the sensing layer, network layer, supporting layer and application layer. Smart technique is applied to the planning, designing, construction, management, updating and other stages of the park green space, so as to provide efficient and convenient services for the government, the designers, the public, management personnel and so on. In conclusion, the application of the smart technology can promote the rationality of the planning and construction, enhance the friendliness of the usage of park green space environment, and improve the management efficiency and scientificity of the park green space.
\end{abstract}

Keywords: Smart technology, Park, Construction and management

\section{Introduction}

As one of the urban ecological infrastructures, urban green space plays an important role in ecosystem services function and value [1]. Urban parks are the main components of urban public green space. Except the ecological functions such as reducing the heat island effect, reducing urban pollution, increasing biodiversity and so on, urban parks are also the places for leisure activities with disaster-prevention function, which are irreplaceable [2]. According to the present classification criteria of the urban green space system in China, urban green space consists of 5 parts, which are park green space, attached green space, green belt, productive green space and other green space. The park green space includes all kinds of parks in the urban built-up area and roadside green space. According to the shapes, the park green space is divided into dot pattern, strip pattern and ribbon pattern: The dot patterns usually represent roadside green space, which has small area but it's the most widely distributed; The strip-shaped ones are mostly parks which are relatively bigger; while the ribbon-shaped ones are the parks that located along roads, drainage systems, city walls and others. The urban park green space is the green space rather frequently used by the public, is an important reference to measure the quality of the public recreation environment in the city, and plays an important social function.

The life cycle of green park space consists of several phases, including planning and designing, construction, management, renewal and so on. During the actual operation process, the relatively great deficiency in information collecting, processing and coordinating may result in various problems in the construction and management of the 
park green space, which affect the performance of the functions of the green space in the park. IT plays an increasingly important role in urban construction, enhancing the efficiency of the urban construction and management. Likewise, IT can also play an important role in the construction and management of the urban park green space.

Since IBM proposed the concept of "Smart Cities", the combination of smart technology and city special project construction has produced a great number of application achievements [3]. The intellectual technology has been widely applied to such fields as smart cities [4], smart communities [5], and smart transportation and so on, improving the operation efficiency. Similarly, the development of the smart technology has provided opportunities for the park green space. By applying the Internet of Things and cloud computing, represented by mobile technology, smart technology can realize overall perception, ubiquitous interconnection, universal computing and fusion application, and it can also make the public openness come true by applying social network. Applying smart technologies to the construction and management of urban park green space has a broad prospect. However, studies on applying smart technologies to park green space are really scarce. This study tried to establish the technical framework of smart technique is applied to the park green space, and conducted a detailed breakdown of application modules.

\section{Method and Application Framework}

\subsection{Method}

The application of smart technologies should meet the requirements of the life cycle and service objects of the park green space [Figure 1]. The life cycle includes stages such as planning and design, construction, management and update, while service objects should include designers, the government, builders, managers, the public etc. In the phase of planning and designing, the designer makes a basic orientation for the park according to the special planning for the green space system, and after having a knowledge of the public demand and the site environment, the designer will make a plan according to the site features. To ensure the rationality of the planning, the designer needs a large amount of data as support, while obtaining of such data often needs coordination with multiple departments, which costs plenty of time. The smart platform can achieve a rapid collecting and processing of information. The construction process is usually coordinated by the investor (the government), constructor and supervisor. As an investor, the government needs to control the park's construction quality and progress and release them to the public timely, while the progress information can be published through the smart platform at any time. Management of the park green space is the stage with the longest duration, and it costs much manpower, material and financial resources. The smart technology can realize the automation of environmental perception and maintenance management, and can achieve the aim of economic saving by increasing the efficiency. Any park green space shall be adjusted and updated according to the public needs and environment change after a term of service. This kind of change is based on the long-term monitoring data. In order to serve the public better, all kinds of information will be made available to the public in time, and easy to use for the public. 


\subsection{Application Framework}

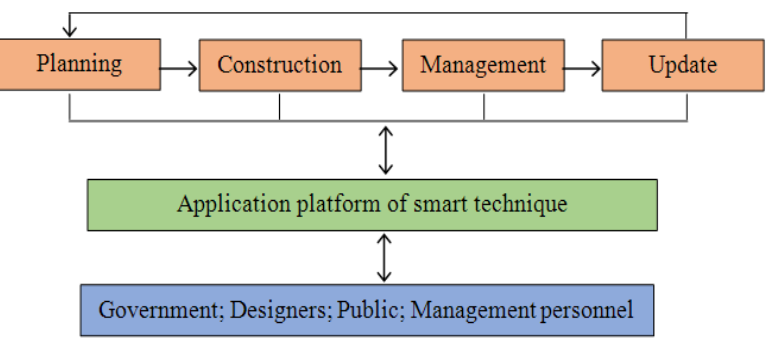

Figure 1. Application Methods of Smart Technique

The smart technology platform aims to build modern smart park green space, and emphasizes improving the monitoring, management and service abilities of the park green space, and synthetically utilizes the integrated technology of spatial information, 3S technology, smart sensing technology, Internet of Things, cloud computing technology and so on to form an overall framework. The framework of the smart park green space is composed of four levels, which are respectively the sensing level, network level, supporting level and application level [Figure 2]. Relying on the various sensor nodes deployed in the parkland, the sensing layer achieves the smart sensing and dynamic monitoring, builds a modern, comprehensive, smart monitoring and management system of the park green space, realizes the real-time perception of the park green space, facilities, tourists, transportation, scenic spots, routes and other type of information, and provides a comprehensive data support for the management of green space. The network layer comprehensively applies the optical fiber, WIFI, the mobile Internet, 3G and many other network technological means to realize a whole multi-network coverage in the park green space. The supporting layer is mainly responsible for the collection and storage of data, intelligent computation and analysis and service management. The application layer provides the technology application of the park green space to different users through the smart platform in a modular manner. Users can carry out convenient operations through such hardware equipment as smart phones, touch devices, computers, and such software applications as APP software, network software.

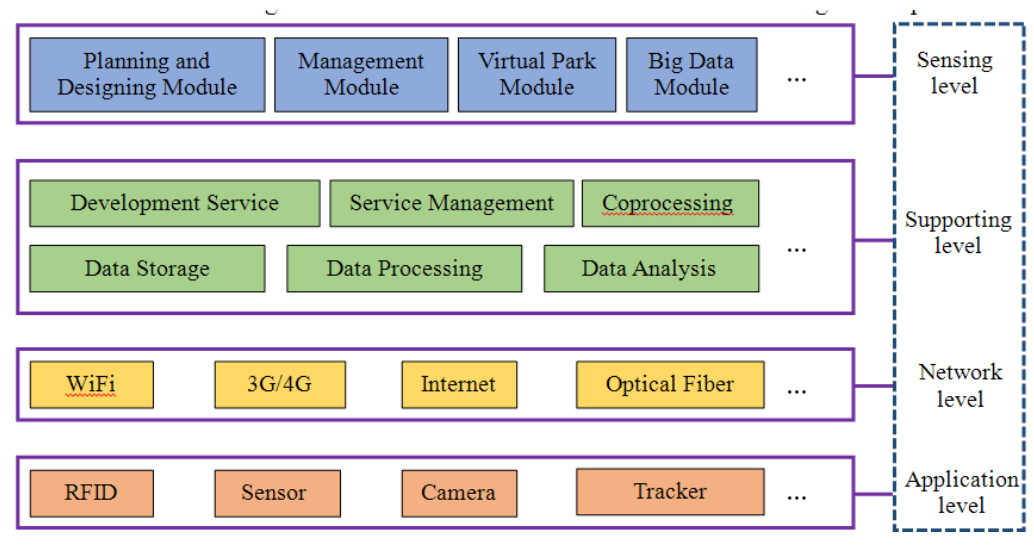

Figure 2. Application Framework of Smart Technique

\section{The Application Module of the Smart Technology}

\subsection{Planning and Designing Module}

3.1.1. Professional Planning Sub-Module: Currently, the landscape planning has been subject to the data acquisition for a long time, and the basic data of planning work highly 
depends on the official survey data, the statistical data and the data from the competent authorities of the industry. Park green space planning needs ecological environment and geographic information, city planning and specialized planning information as well as social information, etc., The completeness and effectiveness of park green space data affect the rationality of planning directly because of the planning's increasing reliance on data. The coordination between specialized planning module and smart urban module helps the collection, transmission and feedback of the information. Understanding the demands of the public by public participation is beneficial to identify and find potential factors that may influence the planning, promote Park green space planning to be more in line with reality and make sure that park green space achieves its anticipated benefits.

3.1.2. Public Participation Planning Sub-Module: Public participation is an important measure to promote the rationality of planning and building. Generally, public participation is that after the designers complete the planning proposal, it is released to the public, and then modified and improved according to public opinions collected. Publicparticipation Planning is different from the previous way of public participation, and it allows the public to participate in the planning and design in the beginning stage of the program. The platform achieves the goal that the planning scheme is completed by both the public and the designers through a simple and easy interface and planning tools. This way can deeply satisfy the public's demands and maximally guarantee the public's interests.

\subsection{Management Module}

3.2.1. RFID Management Sub-Module: The RFID (Radio Frequency Identification) technology is a communication technology which can identify specific target and readwrite relevant data by means of radio signal without building mechanical or optical contact between recognition system and specific target. This technology is simple and easy to use, and it has an extremely high application value in the park green space management, which is mainly, reflected in the guide description, caring for the vulnerable groups, safety monitoring, supervision of rare trees, etc. Information about culture, history, nature and landscape of the park green space is stored in RFID labels and placed along the park green space to for the convenience of the public inquiry. This function can also provide care services for vulnerable groups. RFID labels can also be used in safety monitoring, especially for children. Through the wristbands with labels worn by tourists, the whereabouts of tourists can be tracked, and the specific position can be shown on the screen. Through creating RFID labels for old trees and famous wood species or the ancient buildings, etc., it is convenient for maintenance workers to learn about the information of the maintenance such as the time, the content, the technology.

3.2.2. Green Space Management Sub-Module: Green space management includes the environmental data release, automatic control and security monitoring. Environmental data released mainly by ground sensors provide such real-time data as humidity, temperature, wind speed, ultraviolet ray, suspended particles, negative ions, and noise, thus, the public can view through online map. Automatic control mainly refers to that the smart irrigation system sets parameters to automatically control the irrigation equipment after intelligent analysis based on information such as soil humidity provided by sensors. Security monitoring is to install monitoring equipment in the important or dangerous area of the park to ensure public security.

\subsection{Virtual Park Module}

The 3D simulation park green space system is created based on the GIS technology and 3D modeling technology to provide the public with convenient inquiry, measurement, roaming and viewing in a flying-through mode. The information of the buildings, 
facilities, roads, plants, landscapes, feature spots and activities, etc. of the park green space can be inquired in a real time manner. Virtual emergency and disaster-prevention can guide the evacuation and emergency refuge functions in the park green space, and promote the public's awareness of the emergency and disaster-prevention function of the park.

\subsection{Big Data Module}

The rationality of the construction and management of the park green space is need of scientific evidence. However, because of shortage of effective data, many scientific research projects can't be carried out further. Designers can only carry out the planning relying on their professional experience. However, after its implementation, it is hard to evaluate whether it can meet the demands of the public or not. The big data module is mainly used to collect various information through the following approaches, mutual interconnection among specialized sensors can achieve the fast collection of data; users finish a complicated task collectively by actively sharing the data they have obtained. For example, through active cooperation, questionnaires are completed, or through mobile phones, activities in the park green space are shared through their own mobile phones.

\section{Conclusion}

The park green space is an important place for public leisure and entertainment. To promote the service quality of the park green space, application of the smart technology to the park system is the inevitable tendency of development. The core of the smart technology is to construct the ubiquitous park service with equal opportunities for the public and oriented to the park. The smart technology can realize public on-demand access to information at any time, and enhance the friendliness of the park green space environment. The smart technology helps to achieve the optimal allocation of park resources, thereby reducing the consumption of resources and energies. Through the comprehensive interconnection, intercommunication and interaction between people and objects and that between people and people, the efficiency and scientificity of the management the park green space.

\section{Acknowledgements}

This project is financially supported by the Science and Technology Innovation Fund Project of Young Teachers in Henan Institute of Science and Technology, 2014 (Project title: Research on the Planning and Coordinated Mechanism of Smart Agriculture Science and Technology Demonstration Park) and The Key Scientific Research Project of Universities in Henan Province (Project number is: 15B220001).

\section{References}

[1] I. Middle, P. Dzidic, A. Buckley, D. Bennett, M. Tye and R. Jones, "Integrating community gardens into public parks, an innovative approach for providing ecosystem services in urban areas", Urban Forestry \& Urban Greening, vol. 13, no. 4, pp. 638-645, (2014).

[2] P. Cohen, O. Potchter and I. Schnell, "A methodological approach to the environmental quantitative assessment of urban parks", Applied Geography, vol. 48, (2014), pp. 87-101.

[3] S. Paroutis, M. Bennett, L. Heracleous, "A strategic view on smart city technology: The case of IBM Smarter Cities during a recession", Technological Forecasting and Social Change, vol. 89, (2014), pp. 262-272.

[4] P. Neirotti, A. D. Marco, A. C. Cagliano, G. Mangano and F. Scorrano, "Current trends in Smart City initiatives, Some stylised facts", Cities, vol. 38, no. 25-36, (2014).

[5] Z. Y. Huang, "Evaluating intelligent residential communities using multi-strategic weighting method in China”, Energy and Buildings, vol. 69, (2014), pp. 144-153. 


\section{Authors}

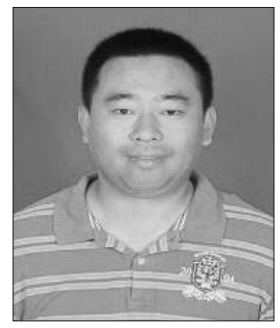

Yichuan Zhang, male, he received a Master degree from Central South University of Forestry and Technology, Changsha, China, in 2008, now he is an associate professor in the School of Horticulture and Landscape Architecture, Henan Institute of Science and Technology, Xinxiang, China. His current research interests include the landscape evaluation and the application of mathematical models in landscape optimization. E-mail: zhangyichuan2002@163.com.

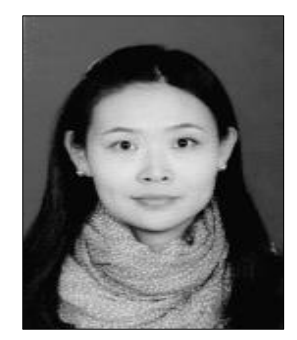

Yan Li, female, she received a Master degree from Zhengzhou University, China, in 2010, now she is a lecture in the Henan Technical College of Construction, China. Her current research interests include the landscape planning and landscape design. E-mail: 177256933@qq.com. 